\title{
A review on morphological evolution of thin metal films on weakly-interacting substrates
}

Kostas Sarakinos

The self-archived postprint version of this journal article is available at Linköping University Institutional Repository (DiVA):

http://urn.kb.se/resolve?urn=urn:nbn:se:liu:diva-160976

N.B.: When citing this work, cite the original publication.

Sarakinos, K., (2019), A review on morphological evolution of thin metal films on weakly-interacting substrates, Thin Solid Films, 688, 137312. https://doi.org/10.1016/j.tsf.2019.05.031

Original publication available at:

https://doi.org/10.1016/j.tsf.2019.05.031

Copyright: Elsevier

http://www.elsevier.com/

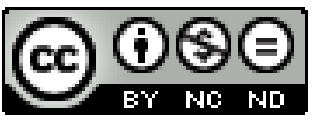




\title{
A review on morphological evolution of thin metal films on weakly-interacting substrates
}

\author{
K. Sarakinos \\ Nanoscale Engineering Division, Department of Physics, Chemistry, and Biology, \\ Linköping University, SE 581 83, Linköping, Sweden \\ e-mail: kostas.sarakinos@liu.se
}

\begin{abstract}
The interaction strength between film-forming species and substrate atoms is a decisive factor in determining film morphological formation and evolution. When the interaction is weak, as e.g., during deposition of thin metal films on oxide and 2Dmaterial substrates, a pronounced 3D morphology is obtained. Owing to the great technological relevance of these film/substrate systems, the present paper reviews theories and recent developments with regards to the film growth dynamics and the atomistic origin of 3D film morphology. It also highlights possible future directions toward which this sub-field of thin film science and technology can develop.
\end{abstract}

\section{Introduction}

The effect of thin films on the properties of surfaces upon which they are deposited depends, crucially, on the film morphology [1,2]. An important factor in determining film morphology is the interaction strength between film-forming species and substrate atoms, as described in the classical work of Bauer [3], who used surface free-energy minimization arguments for film $\left(\gamma_{f}\right)$ and substrate $\left(\gamma_{s}\right)$, to suggest that: (i) a two- 
dimensional (2D) morphology is obtained when film and substrate interaction is strong, such that $\gamma_{s}>\gamma_{f}+\gamma_{\text {int }}\left(\gamma_{\text {int }}\right.$ is the film/substrate interface energy); and (ii) a threedimensional (3D) morphology is obtained when $\gamma_{s}<\gamma_{f}+\gamma_{\text {int }}$, i.e., in the case of weakly-interacting film/substrate systems (see Fig. 1 for a schematic illustration). Even though Bauer's theoretical framework was originally developed to describe epitaxial growth, it has been widely used for classifying morphology of polycrystalline films, since local epitaxial growth conditions may prevail at the length scale of individual grains $[2,4,5]$.

Typical examples of weakly-interacting film/substrate systems include metal layers growing on oxide and 2D-material (e.g., graphene) surfaces [6,7]. The 3D film growth character in these systems is frequently leveraged for synthesis of supported nanoparticles and nanostructures for catalytic applications [6]. Concurrently, the pronounced tendency toward 3D morphology can be a drawback for applications in which growth of flat metal layers is required, as, e.g., in low-emissivity energy-saving windows [8], optoelectronic devices [9,10], biosensors [11,12], and nanoelectronic components $[7,13,14]$. Hence, a fundamental understanding of the mechanisms that govern thin metal film morphological evolution on weakly-interacting substrates is a prerequisite for directed growth of metal layers and nanostructures with controlled shape, microstructure, and interface properties in high-performance devices founded upon oxides and 2D materials.

A large fraction of thin films is today synthesized via vapor-based deposition [1,2]. As such, vapor condensation and film growth proceed far from equilibrium $[1,2]$ and the resulting film morphology is primarily determined by the relative rates of competing 
atomistic structure-forming processes (i.e., by kinetics) $[1,2,4]$. Hence, the Bauer classification, although it paints a general picture of the expected film microstructure in view of the film/substrate interaction strength, it does not provide access to an in-depth understanding of the atomic-scale processes that control film morphological evolution.

Currently, the most detailed atomistic description of far-from-equilibrium 3D island formation is based on homoepitaxial systems in which 3D islands (mounds) form by deposition onto existing small islands, followed by atomic-step descent limited by the Ehlrich-Schwöbel barrier [15]. However, for weakly-interacting film/substrate systems 3D islands develop before the initially-formed one-atom-high islands are large enough to efficiently capture vapor-phase deposition flux [7, 16-24]. The latter highlights that a different framework-as compared to standard homoepitaxial growth theory-is needed for understanding, on a fundamental level, film morphological evolution on weakly-interacting substrates.

The present paper reviews, briefly, established theories and recent developments with respect to thin metal film growth on weakly-interacting substrates, with the goal to offer a quick first overview of the field to the interested reader. It starts with a description of film growth stages and a discussion of the way by which dynamic competition among structure-forming processes leads to characteristic morphological transitions (Section 2). The atomic-scale processes that drive 3D morphology formation and evolution, and the corresponding theoretical framework are discussed in Section 3. The paper is summarized in Section 4, which also highlights possible future research directions in the field. 


\section{Dynamics of thin-film growth stages and morphological transitions}

Growth of polycrystalline films starts with vapor condensation on the substrate surface and formation of spatially separated single-crystalline islands (nucleation), which grow in size (island growth) and impinge on each other forming new larger islands (coalescence). Reshaping of impinging islands and coalescence necessitate that surface and bulk atoms are mobile, i.e., they occur at kinetic conditions that correspond to the range covered by zones $\mathrm{T}$ and/or 2 of the classical structure zone model (SZM) diagram [4,5]. When these conditions are not fulfilled, i.e., growth occurs in zone 1 of the SZM [4,5], coalescence is not completed throughout all film-formation stages, as discussed in detail in Section 2.2. The process of coalescence also leads to a reduction of the island density on the substrate surface and continues until the boundaries between single-crystalline islands (i.e., grains) become immobile and coalescence stops. Subsequent deposition fills with material the inter-island space (hole filling) and, once this process is completed, a continuous film is formed. These growth stages are particularly distinct in weakly-interacting film/substrate systems that exhibit a pronounced 3D morphology, as shown for the case of magnetron-sputtered Ag films deposited on $\mathrm{SiO}_{2}$ substrates in Fig. 2. During the various film growth stages, competing structure-forming processes lead to characteristic morphological transitions, as explained in the following and indicated in Fig. 2.

\subsection{Island nucleation vs. island growth and saturation island density}

From an atomistic point of view, film-forming species from the vapor phase (i.e., atoms and/or molecules) are subjected to an attractive potential, once in the vicinity of the substrate, and they are accelerated gaining kinetic energy, which is of the order of the cohesive energy of the substrate lattice. The gained kinetic energy is dissipated, upon 
impingement on the substrate surface, into crystal lattice vibrations (phonons) causing vapor species to adsorb on the substrate, i.e., to become adspecies (adatoms and/or admolecules)

At finite temperatures, adspecies perform a thermally activated two-dimensional random walk on the substrate, executing jumps between neighboring adsorption sites, as determined by the potential-energy landscape of the surface. The rate of successful jumps and, thereby, the distance travelled by adspecies during this walk, is expressed in terms of their diffusivity:

$$
D=1 / 4 l^{2} v
$$

In Eq. (1) $l^{2}$ denotes the mean square displacement covered per single jump, and $v$ is the diffusion rate which is equal to

$$
v=v_{0} e^{-E_{D} / k_{B} T}
$$

where $T$ is the substrate temperature, $k_{B}$ denotes the Boltzmann's constant, $E_{D}$ is the energy barrier that an adatom must overcome for the jump between adjacent sites to occur, and $v_{0}$ is the attempt frequency. Typical values for $v_{0}$ are of the order $10^{12}-10^{13}$ $\mathrm{s}^{-1}$, while $E_{D}$ can vary in the range 0.05 to $1.0 \mathrm{eV}$ or even be higher [15].

Vapor deposition increases the adspecies density on the substrate surface until adspecies-adspecies encounters become inevitable and clusters (islands) form; a process that is referred to as nucleation. Islands may dissociate or grow in size by incorporating adspecies and/or material from the vapor phase $[25,26]$. In general, islands may also be mobile with their mobility depending on their size and chemical nature [27]. The process of nucleation results in an increase of the island density $N$ 
on the substrate, until a saturation value $N_{\text {sat }}$ is reached, when the island formation rate becomes equal to the rate of adspecies incorporation into existing islands. In the simplest scenario, adatoms are the only mobile species on the substrate surface and dimers are stable (referred to as irreversible nucleation). In that case, $N_{\text {sat }}$ is expressed as [28]:

$$
N_{s a t} \propto\left(\frac{F}{D}\right)^{x}
$$

In Eq. (3), $x$ is a scaling exponent smaller than one that depends on the dimensionality of the growth (i.e, 2D or 3D growth) and the critical nucleus size (i.e., the size of the smallest stable atomic cluster), and $F$ is the adatom arrival rate (i.e., deposition rate) expressed in monolayers (ML) per second. For 3D islands on a $2 \mathrm{D}$ surface, and for growth conditions that lead to irreversible nucleation, $x=2 / 7$. Equation (3) encodes the dynamic competition among island nucleation and growth during initial filmformation stages. Increase of $D$ (e.g., caused by increasing $T$ ) leads to larger adatom mean free path on the substrate surface. This favors adatom incorporation into existing islands, at the expense of nucleating new ones, and it results in a decrease of $N_{s a t}$ Conversely, increase of $F$ leads to a larger adatom density on the substrate surface. This increases the probability of adatom-adatom encounters and, hence promotes island nucleation at the expense of island growth, resulting in a larger $N_{\text {sat }}$.

\subsection{Island growth vs. island coalescence and elongation transition}

As stated at the outset of Section 2, islands grow larger by incorporation of adspecies and/or material from the vapor phase. Past the point of $N_{s a t}$, island growth becomes the main process that determines the film morphology by increasing the fraction of 
substrate surface covered by the deposit. This is until two or more islands impinge and coalesce into a larger single-crystalline island, which reduces the island density, while erasing morphological features attained during earlier stages of the film growth. A simple way to describe the dynamics of island coalescence is based on the sintering theory developed by Nichols and Mullins [29,30], which assumes that coalescence proceeds by atomic surface diffusion from the convex to the concave area of a coalescing island pair. The time from impingement to full shape equilibration is given by

$$
\tau_{\text {coal }}=R^{4} / B
$$

where $R$ is the radius of the smaller island, and $B$ is the so-called coalescence strength, which is a material- and temperature-dependent constant $[29,30]$.

According to Eq. (4), as $R$ increases $\tau_{\text {coal }}$ increases as well, and there is a critical $R$ value at which $\tau_{\text {coal }}$ becomes longer than the time required for a third island to impinge on a coalescing island pair. This point during growth corresponds to the so-called elongation transition, beyond which the film surface consists predominantly of elongated non-coalesced groups of islands. Analytical modelling, based on the droplet growth theory [31,32], and kinetic Monte Carlo simulations [33-36] suggest that, for film materials and deposition parameters for which coalescence is the dominant process during early stages of film growth (coalescence-controlled growth regime), the nominal film thickness at the elongation transition $\Theta_{\text {elong }}$ scales with $F$ as

$$
\Theta_{\text {elong }} \sim\left(\frac{B}{F}\right)^{\frac{1}{3}}
$$


Equation (5) reflects the effect of dynamic competition among island growth and coalescence on film morphological evolution. For a constant coalescence-rate parameter $B$, increase of $F$ yields a larger island growth rate, such that an elongated surface morphology is attained at smaller nominal thicknesses. Conversely, increase of $B$, at a constant $F$, promotes coalescence completion relative to island growth, thereby delaying the occurrence of elongation transition.

For a given film/substrate system, there are deposition conditions in terms of $F$ and $T$, for which coalescence is not completed throughout all stages of growth (coalescencefree growth regime) $[33,37]$. In this case, $\Theta_{\text {elong }}$ becomes proportional to the islandisland separation distance when island density reaches $N_{\text {sat }}$ [33,38], i.e., $\Theta_{\text {elong }} \sim N_{\text {sat }}{ }^{-\frac{1}{2}}$. Using Eq. (3) for 3D growth the following expression is obtained:

$$
\Theta_{\text {elong }} \sim\left(\frac{D}{F}\right)^{\frac{1}{7}}
$$

In analogy to coalescence-controlled growth (Eq. (5)), Eq. (6) represents the way by which the interplay among island nucleation and growth affects the early-stage film morphology. Increase of $D$, for a given $F$, favors growth of existing islands, at the expense of nucleation of new ones, resulting in an increase of the nominal thickness required for the onset of island-island impingement. In the opposite case, larger $F$, at a constant $D$, promotes nucleation, pushing elongation to occur at smaller nominal thicknesses. 


\subsection{Percolation transition and continuous film formation}

The onset of elongation transition leads to a film surface that is covered, predominantly, by polycrystalline islands. The shapes of grain boundaries in these islands change continuously as result of the competition between boundary and surface energies, while grain boundaries can be mobile, depending on the growth temperature and the grain size [2,4]. These effects lead to grain growth, which in combination with the kinetically controlled rate at which adatoms descend from the surface of the film to the grain boundary base (hole filling) leads to a formation of an interconnected island network (percolation transition) and eventually to a continuous film. Intuitively, one would expect that the nominal thickness at which percolation transition occurs $\left(\Theta_{\text {perc }}\right)$ and a continuous film is formed $\left(\Theta_{\text {cont }}\right)$ are affected by the rates of hole filling and vs. out-of-plane film growth. However, the influence of initial growth stages (island nucleation, growth, and coalescence) on the values and scaling behavior of those thicknesses is very pronounced, as explained in Section 2.4.

\subsection{Experimental determination of morphological transition thicknesses}

The absolute values of $\Theta_{\text {elong }}$ for a given set of synthesis conditions reflect the degree of 3D clustering during growth, whereby larger transition thicknesses indicate a more pronounced 3D morphology. Concurrently, the scaling behavior as function of the deposition rate $F$, describe the relative importance of nucleation vs. coalescence for film morphological evolution [36]. The elongation transition is an intrinsically abstract concept, i.e., $\Theta_{\text {elong }}$ is difficult to determine experimentally [39]. Hence, the scaling behavior in view of Eqs. (5) and (6) is, typically, studied using $\Theta_{\text {perc }}$ and $\Theta_{\text {cont }}$, which have been shown to scale linearly with $\Theta_{\text {elong }}[34,39]$. This was first shown by 
Warrender and Aziz [18] who measured (using in situ resistivity) $\Theta_{\text {perc }}$ during pulsed laser deposition of $\mathrm{Ag}$ films on $\mathrm{SiO}_{2}$ substrates and established a $\Theta_{\text {perc }} \sim(1 / F)^{1 / 3}$ scaling law. Later Elofsson et al. [19] and Lü et al. [35] measured $\Theta_{\text {perc }}$ and $\Theta_{\text {cont }}$ during growth of $\mathrm{Ag}$ on $\mathrm{SiO}_{2}$ by continuous and pulsed magnetron sputtering, using in situ spectroscopic ellipsometry. They found that these thicknesses scale as $\left(\frac{1}{F}\right)^{\frac{1}{7}}$ for room-temperature deposition, while a transition towards coalescence-controlled growth $\left((1 / F)^{1 / 3}\right.$ scaling behavior) is observed at elevated temperatures (see Fig. 3 for a set of representative data). These results confirmed the theoretical framework that was used to derive Eqs. (5) and (6). They also enabled estimation of atomic-scale diffusion rates during growth of $\mathrm{Ag}$ and $\mathrm{Cu}$ films on $\mathrm{SiO}_{2}$ and amorphous carbon substrates by studying the scaling behavior of $\Theta_{\text {perc }}$ and $\Theta_{\text {cont }}$ vs. $F$ at various growth temperatures $[39,40]$.

\section{Atomic-scale view on 3D morphological formation and evolution}

Another important aspect, besides the description of metal-film growth dynamics on weakly-interacting substrates, is the identification of atomistic processes that drive 3D morphological formation and evolution. As explained in Section 1, the most established theoretical framework for explaining 3D film growth is based on the categorization proposed by Bauer [3]. Following this, recent studies of metal-film and nanostructure growth on graphite [41], graphene [7], MoS $2[13,14]$, and oxides [6] have explained experimentally-observed morphologies as a function of the ratio between the adsorption energy $\left(E_{\text {ads }}\right)$ of metal atoms on the substrate and the bulk-metal cohesive energy $\left(E_{c o h}\right)$; the lower the $E_{\text {ads }} / E_{c o h}$ ratio, the stronger the tendency toward 3D 
growth. Moreover, the absolute values of the energies set the kinetics of morphological evolution, e.g., the lower the $E_{a d s}$ value, the lower the temperature at which transition from 2D to 3D growth morphology occurs. Both Bauer classification, which is based on surface free-energy minimization, and the $E_{a d s} / E_{c o h}$ criterion provide insights into the energetic origin of 3D growth. In order to obtain a full picture of the mechanisms that control interlayer mass transport and 3D island formation and evolution a kinetic description of the atomic-scale pathways is required, as explained in the following.

Although film morphology is determined by a multitude of mechanisms throughout the growth stages shown in Fig. 2, the first obvious step is to attempt to decipher shape evolution of individual 3D islands. Intuitively, the tendency toward 3D growth in weaklyinteracting film/substrate systems can be understood as the metal atoms being bound weaker to the substrate (relative to themselves), which facilitates their ascent onto the first layer of an island (process (1) in Fig. 4(a)). However, beyond the first atomic layer, growth is primarily homoepitaxial, and thus governed once again by negligible step ascent ((process (2) in Fig. 4(a)). Thus, weak adatom/substrate bonds (manifested by low $E_{\text {ads }} / E_{c o h}$ ratios or the condition $\gamma_{s}<\gamma_{f}+\gamma_{\text {int }}$ ) can only explain the initial 3D island formation, but not the way by which the island shape evolves with continued deposition. Campbell [6] suggested that continued growth of 3D islands on weaklyinteracting substrates, beyond the second atomic layer, occurs due to assisted upstepping. This mechanism assumes a reduction in the step ascent barrier, when adjacent layers are separated horizontally by only one atom, due to an attractive force from the upper step edge (process (3) in Fig. 4(a)). Modification of step-edge barriers due to short-range interactions has also been proposed to be relevant for facilitating 
the concerted exchange of atoms toward the upper layer of atomic islands in homoepitaxial systems (e.g., $\mathrm{Cu} / \mathrm{Cu}(111)$ and $\mathrm{Ag} / \mathrm{Ag}(100))[42,43]$.

An alternative route to $3 \mathrm{D}$ island growth is associated with on the formation of lowindex sidewall facets, rather than stepped-structures (Fig. 4(b)). Barna et al. [5,44,45] suggested that these facets facilitate growth of AISn alloy films on weakly-interacting substrates_-including amorphous- $\mathrm{C}, \mathrm{SiO}_{2}, \mathrm{NaCl}$, and mica-by providing pathways for accelerated mass transport between the base and the upper layer of 3D atomic islands. Moreover, facet formation has been experimentally observed during the deposition of metal films on graphene and graphite surfaces $[7,41]$. Hence, in the context of 3D growth beyond the second layer, the absence of steps may allow adatoms to ascend multiple layers by encountering a terrace diffusion barrier that is much smaller than its corresponding step-ascent counterpart (process (1) in Fig. 4(b) vs. processes (1) through (3) in Fig. 4(a)). The final 3D island shape would then be controlled by the rates of atom diffusion on the facets and facet/facet crossing (process (2) in Fig. 4(b)), rather than the rate of step-edge crossing [46-48]. The formation of side wall facets and their role for 3D island shape evolution was recently confirmed by Lü et al. [49] and Gervilla et al. [50] using kinetic Monte-Carlo simulations and analytical modelling. Their results showed that $3 \mathrm{D}$ nuclei are first formed due to facile adatom ascent at single-layer island steps, followed by the development of sidewall facets bounding the islands, which in turn facilitates upward diffusion from the base to the top of the islands. The limiting atomic process which determines the island height, for a given number of deposited atoms, is the temperature-dependent rate at which adatoms cross from sidewall facets to the island top. Hence, increase of temperature leads to increase of island height $[49,50]$; this is opposite to homo-epitaxial growth theory and simulations 
[15], which predict that increase of temperature enhances the downward interlayer atomic transport and leads to flatter islands. These results are consistent with previous experimental observations which show that increasing growth temperatures during metal deposition on weakly-interacting substrates-including $\mathrm{Ag} / \mathrm{SiO}_{2}[18,19,35,40]$, Ag/graphene [51], $\mathrm{Cu} / \mathrm{ZnO}$ [22,23], $\mathrm{Pd} / \mathrm{TiO}_{2}$ [21], and Dy/graphene [24]—gives rise to an increase in surface roughness. Instead, increase of growth temperature in homoepitaxial film/substrate systems lead to smoother film surfaces [15,52], see e.g., Fig. 5 for a comparison between Ag/graphene and Ag/Ag(111) systems.

As final note, it should be emphasized that atom-by-atom diffusion is not the only mechanism that drives interlayer mass transport. Collective atomic motion, which has been reported to occur in metal-on-metal and metal-on-semiconductor film/substrate systems $[42,43,53]$, can be a possible pathway for explaining formation of 3D metal islands on weakly-interacting substrates.

\section{Summary and outlook}

This paper presents a brief review with regards to the current understanding of film growth on weakly-interacting substrates. Weak bond strength among film-forming species and substrate atoms leads to formation of 3D nanostructures and as the film grows thicker its morphology undergoes characteristic transitions (elongation, percolation, and continuous film formation). Theoretical models, simulations, and experimental data show that the thickness at which these transitions occur exhibit distinct scaling laws as function of deposition temperature and rate, which reflect the dynamic competition among structure structure-forming processes throughout the different growth film growth stages. 
3D morphological formation and evolution of thin films on weakly-interacting substrates is well-understood from a thermodynamic point of view, but less is known with regards to its atomistic origin. Classical homoepitaxial theory, in which mound formation and film roughening is ascribed to limited downward interlayer transport due to the stepedge-descend barrier, cannot explaining formation and shape evolution of 3D islands. There is a wide agreement across the literature that $3 \mathrm{D}$ island formation on weaklyinteracting substrates is driven by upward mass transport from the substrate to the island top. A combination of experimental and simulation data indicate that this is likely to be mediated by formation of faceted islands that offer accelerated upward atomic diffusion direction. The rate of such diffusion is enhanced with increasing growth temperature, which can explain the formation of rougher film surface at elevated temperatures observed in many weakly-interacting film/substrate systems.

Although weakly-interacting film/substrate systems are widely used in a gamut of applications, the fundamental understanding of atomic-scale processes that govern film morphological evolution is still at its infancy, as compared to homoepitaxial film/substrate systems. Such understanding can be expanded by using multi-scale modelling (e.g., by combining KMC simulations with classical and ab initio Molecular Dynamics) to describe growth stages beyond single island formation, including coalescence and hole-filling. Theory should be combined atomic-scale studies using e.g., in situ microscopy techniques in order to link theoretical predictions with experimental observations. This would generate key knowledge and open the way for depositing, e.g., metals with controlled morphologies on a number of technologicallyrelevant weakly-interacting substrates. 


\section{Acknowledgements}

I am grateful to Dr. Viktor Elofsson, Mr. Andreas Jamnig, Mr. Victor Gervilla, Dr. Bo Lü, and Dr. Daniel Magnfält (names listed in alphabetical order) whose contributions have been instrumental for expanding our understanding of morphological evolution in weakly-interacting film/substrate systems. Moreover, over the past 10 years, I have benefited tremendously by close interactions and collaboration with Prof. Gregory Abadias (University of Poitiers, France) and Dr. Davide Sangiovanni (Linköping University, Sweden).

\section{References}

[1] M. Ohring, Materials Science of Thin Films, Academic Press, San Diego, 1991.

[2] P. M. Martin, Handbook of Deposition Technologies for Films and Coatings, Elsevier, Amsterdam, 2005.

[3] E. Bauer, Phänomenologische Theorie der Kristallabscheidung an Oberflächen. I, Z. Kristallogr. 110 (1958) 372-394. https://doi.org/10.1524/zkri.1958.110.1-6.372. [4] I. Petrov, P.B. Barna, L. Hultman, and J.E. Greene, Microstructural evolution during film growth, J. Vac. Sci. Technol. A $21 \quad$ (2003) S117-S128. https://doi.org/10.1116/1.1601610.

[5] P.B. Barna and M. Adamik, Fundamental structure forming phenomena of polycrystalline films and the structure zone models, Thin Solid Films 317 (1998) 27-33. https://doi.org/10.1016/S0040-6090(97)00503-8.

[6] C.T. Campbell, Metal films and particles on oxide surfaces: structural, electronic and chemisorptive properties, J. Chem. Soc. Faraday Trans. 92 (1996) 1435-1445. https://doi.org/10.1039/FT9969201435.

[7] X. Liu, Y. Han, J.W. Evans, A.K. Engstfeld, R.J. Behm, M.C. Tringides, M. Hupalo, 
H.Q. Lin, L. Huang, K.-M Ho, D. Appy, P.A. Thiel, and C.-Z. Wang, Growth morphology and properties of metals on graphene, Prog. Surf. Sci. 90 (2015) 397-443. https://doi.org/10.1016/j.progsurf.2015.07.001.

[8] C. Schaefer, G. Bräuer, and J. Szczyrbowski, Low emissivity coatings on architectural glass, Surf. Coat. Technol. $93 \quad$ (1997) 37-45. https://doi.org/10.1016/S0257-8972(97)00034-0

[9] T.J. Echtermeyer, S. Milana, U. Sassi, A. Eiden, M. Wu, E. Lidorikis, and A.C. Ferrari, Surface Plasmon Polariton Graphene Photodetectors, Nanolett. 16 (2016) 820. https://doi.org/10.1021/acs.nanolett.5b02051.

[10] T. Mueller, F. Xia, and P. Avouris, Graphene photodetectors for high-speed optical $\begin{array}{lllll}\text { communications, } & \text { Nat. } & \text { Phot. } & 4 & \text { (2010) }\end{array}$ https://doi.org/10.1038/nphoton.2010.40.

[11] Xu, Y., Hsieh, C.-Y., Wu, L., and Ang, L.K., 2019. Two-dimensional transition metal dichalcogenides mediated long range surface plasmon resonance biosensors, J. Phys. D: Appl. Phys. 52, 065101. https://doi.org/10.1088/1361-6463/aaf0f7.

[12] F.H.L. Koppens, D.E. Chang, and F.J.G. de Abajo, Graphene Plasmonics: A Platform for Strong Light-Matter Interactions, NanoLett. 11 (2011) 33703377. https://doi.org/10.1021/nl201771h.

[13] C. Gong, C. Huang, J. Miller, L. Cheng, Y. Hao, D. Cobden, J. Kim, R.S. Ruoff, R.M. Wallace, K. Cho, X. Xu, and Y. J. Chabal., Metal Contacts on Physical Vapor Deposited Monolayer MoS 2 , ACS Nano $7 \quad$ (2013) $11350-11357$. https://doi.org/10.1021/nn4052138.

[14] W.A. Saidi, Trends in the Adsorption and Growth Morphology of Metals on the $\operatorname{MoS}_{2}(001)$ Surface, Crystal Growth and Design 15 (2015) 3190-3200. https://doi.org/10.1021/acs.cgd.5b00269. 
[15] T. Michely and J. Krug, Islands, Mounds, and Atoms: Patterns and Processes in Crystal Growth Far from Equilibrium, Springer, Berlin,2003.

[16] E. Byon, T.W.H. Oates, and A. Anders, 4, Coalescence of nanometer silver islands on oxides grown by filtered cathodic arc deposition, Appl. Phys. Lett. 82 (2003) 16341636. https://doi.org/10.1063/1.1558955.

[17] H.C. Kim, T.L. Alford, and D.R. Allee, Thickness dependence on the thermal stability of silver thin films, Appl. Phys. Lett. 81 (2002) 4287-4289. https://doi.org/10.1063/1.1525070.

[18] Warrender, J. and Aziz, M., 2007. Effect of deposition rate on morphology evolution of metal-on-insulator films grown by pulsed laser deposition, Phys. Rev. B 76, 45414. https://doi.org/10.1103/PhysRevB.76.045414.

[19] Elofsson, V., Lü, B., Magnfält, D., Münger, E.P., and Sarakinos, K., 2014. Unravelling the physical mechanisms that determine microstructural evolution of ultrathin Volmer-Weber films, J. Appl. Phys. 116, 044302. https://doi.org/10.1063/1.4890522.

[20] Krishna, H., Sachan, R., Strader, Favazza, J.C., Khenner, M., and Kalyanaraman, R., 2010. Thickness-dependent spontaneous dewetting morphology of ultrathin Ag films Nanotechnology 21, 155601. https://doi.org/10.1088/0957-4484/21/15/155601.

[21] M.J.J. Jak, C. Konstapel, A. van Kreuningen, J. Verhoeven, and J.W.M. Frenken, Scanning tunnelling microscopy study of the growth of small palladium particles on TiO2(110), Surf. Sci. 457 (2000) 295-310. https://doi.org/10.1016/S0039$\underline{6028(00) 00431-3 .}$

[22] K.H. Ernst, A. Ludviksson, R. Zhang, J. Yoshihara, and C.T. Campbell, Growth model for metal films on oxide surfaces: Cu on $\mathrm{ZnO}(0001)-\mathrm{O}$, Phys. Rev. B 47 (1993) 13782-13796. https://doi.org/10.1103/PhysRevB.47.13782. 
[23] J. Yoshihara, S.C. Parker, and C.T. Campbell, Island growth kinetics during vapor deposition of $\mathrm{Cu}$ onto the Zn-terminated $\mathrm{ZnO}(0001)$ surface, Surf. Sci. 439 (1999) 153162. https://doi.org/10.1016/S0039-6028(99)00752-9.

[24] D.McDougall, H.Hattab, M.T.Hershberger, M.Hupalo, M. Horn von Hoegen, P.A.Thiel, and M.C.Tringides, Dy uniform film morphologies on graphene studied with SPA-LEED and STM, Carbon $108 \quad$ (2016) 283-290. https://doi.org/10.1016/i.carbon.2016.06.083

[25] J. Frenkel, Theorie der Adsorption und verwandter Erscheinungen, Zeitschrift für Physik 26 (1924) 117-138.

[26] G. Zinsmeister, A contribution to Frenkel's theory of condensation, Vacuum. 16 (1966) 529-535. https://doi.org/10.1016/0042-207X(66)90349-6.

[27] Sangiovanni, D., Edström, D., Hultman, L., Chirita, V., Petrov I., and Greene, J., 2012. Dynamics of Ti, N, and TiNx (x=1-3) admolecule transport on $\mathrm{TiN}(001)$ surfaces, Phys. Rev. B. 86, 155443. https://doi.org/10.1103/PhysRevB.86.155443.

[28] Kryukov Y., and Amar, J., 2010. Scaling of the island density and island-size distribution in irreversible submonolayer growth of three-dimensional islands, Phys. Rev. B 81, 165435. https://doi.org/10.1103/PhysRevB.81.165435.

[29] F. A. Nichols and W. Mullins, Morphological Changes of a Surface of Revolution due to Capillarity-Induced Surface Diffusion, J. Appl. Phys. 36 (1965) 1826-1835. https://doi.org/10.1063/1.1714360.

[30] F. A. Nichols, Coalescence of Two Spheres by Surface Diffusion, J. Appl. Phys. 37 (1966) 2805-2808. https://doi.org/10.1063/1.1782127.

[31] F. Family and P. Meakin, Scaling of the Droplet-Size Distribution in VaporDeposited Thin Films, Phys. Rev. Lett. $61 \quad$ (1988) 428-432. https://doi.org/10.1103/PhysRevLett.61.428. 
[32] P. Jensen, Growth of nanostructures by cluster deposition: Experiments and simple models, Rev. Mod. Phys. $71 \quad$ (1999) 1965-1735. https://doi.org/10.1103/RevModPhys.71.1695.

[33] Carrey, J. and Maurice, J.-L., 2001.Transition from droplet growth to percolation: Monte Carlo simulations and an analytical model, Phys. Rev. B 63, 245408. https://doi.org/10.1103/PhysRevB.63.245408.

[34] Carrey, J., Maurice, J.-L., 2002. Scaling laws near percolation during threedimensional cluster growth: A Monte Carlo study, Phys. Rev. B 65, 205401. https://doi.org/10.1103/PhysRevB.65.205401.

[35] Lü, B. , Elofsson, V., Münger, E.P., and Sarakinos, K., 2014. Dynamic competition between island growth and coalescence in metal-on-insulator deposition, Appl. Phys. Lett. 105, 163107. https://doi.org/10.1063/1.4900575.

[36] Lü, B., Münger, E.P., and Sarakinos, K., 2015. Coalescence-controlled and coalescence-free growth regimes during deposition of pulsed metal vapor fluxes on insulating surfaces, J. Appl. Phys. 117, 134304. https://doi.org/10.1063/1.4916983.

[37] P. Jensen, N. Combe, H. Larralde, J. L. Barrat, C. Misbah, and A. Pimpinelli, Kinetics of shape equilibration for two dimensional islands, Eur. Phys. J. B 497 (1999) 497-504. https://doi.org/10.1007/s100510050961.

[38] P. Jensen, H. Larralde, M. Meunier, and A. Pimpinelli, Growth of three-dimensional structures by atomic deposition on surfaces containing defects: simulations and theory, Surf. Sci. 412 (1998) 458-476. https://doi.org/10.1016/S0039-6028(98)00466-X.

[39] Lü, B., Souqui, L., Elofsson, V., and Sarakinos, K., 2017. Scaling of elongation transition thickness during thin-film growth on weakly interacting substrates, Appl. Phys. Lett. 111, 084101. https://doi.org/10.1063/1.4993252. ]

[40] A. Jamnig, D.G. Sangiovanni, G. Abadias, and K. Sarakinos, under review in Sci. 
Rep. (2019).

[41] D. Appy, H. Lei, C.-Z. Want, M.C. Trignides, D.-J. Liu, J.W. Ewans, and P.A. Thiel, Transition metals on the (0001) surface of graphite: Fundamental aspects of adsorption, diffusion, and morphology, Prog. Surf. Sci. 89 (2014) 219-238. https://doi.org/10.1016/i.progsurf.2014.08.001.

[42] J.G. Amar and F. Family, Critical Cluster Size: Island Morphology and Size Distribution in Submonolayer Epitaxial Growth, Phys. Rev. Lett. 74 (1995) 2066-2069. https://doi.org/10.1103/PhysRevLett.74.2066.

[43] Shim, Y. and Amar, J.G., 2011. Ab initio study of edge smoothing, atom attraction, and downward funneling in $\mathrm{Ag} / \mathrm{Ag}(100)$, Phys. Rev. B 83, 245419. https://doi.org/10.1103/PhysRevB.83.245419.

[44] P.B. Barna, G. Barcza, L. Tóth, G. Vincze, A. Bergauer, and H. Bangert, Structural evolution in codeposited AI-Sn thin films, Surf. Coat. Technol. 57 (1993) 7-11. https://doi.org/10.1016/0257-8972(93)90330-Q.

[45] P.B. Barna and M. Adamik, Growth Mechanisms of Polycrystalline Thin Films, In Science and Technology of Thin Films, F.C. Matacotta (Ed), World Scientific Publishing, 1995, pp. 1-28.

[46] Zhang, R. and Huang, H., 2011. Another kinetic mechanism of stabilizing multiplelayer surface steps, Appl. Phys. Lett. 98, 221903. https://doi.org/10.1063/1.3595938. [47] M.G. Lagally and Z. Zhang, Thin-film cliffhanger, Nature 417 (2002) 907-909. https://doi.org/10.1038/417907a.

[48] Y. Han, G.-H. Lu, B.-J. Lee, and F. Liu, Flat-surface, step-edge, facet-facet, and facet-step diffusion barriers in growth of a Pb mesa, Surf. Sci. 602 (2008) 2284-2294. https://doi.org/10.1016/j.susc.2008.05.009.

[49] Lü, B., Almyras, G.A., Gervilla, V., Greene, J.E., and Sarakinos, K., 2018. 
Formation and morphological evolution of self-similar 3D nanostructures on weakly $\begin{array}{lllll}\text { interacting substrates, } & \text { Phys. Rev. Mat. 2, } 063401 .\end{array}$ https://doi.org/10.1103/PhysRevMaterials.2.063401.

[50] V. Gervilla, G.A. Almyras, F. Thunström, J.E. Greene, and K. Sarakinos, Dynamics of 3D-island growth on weakly-interacting substrates, under review in Appl. Surf. Sci. (2019).

[51] H. Zhou, C. Qiu, F. Yu, H. Yang, M. Chen, L. Hu, and L. Sun, ThicknessDependent Morphologies and Surface-Enhanced Raman Scattering of Ag Deposited on n-Layer Graphenes, J. Phys. Chem. 115 (2011) 11348-11354. https://doi.org/10.1021/ip112421q.

[52] K. Bromann, H. Brune, H. Röder, and K. Kern, Interlayer Mass Transport in Homoepitaxial and Heteroepitaxial Metal Growth, Phys. Rev. Lett. 75 (1995) 677-681. https://doi.org/10.1103/PhysRevLett.75.677.

[53] Hersberger, M.T., Hupalo, M., Thiel, P.A., Wang, C.Z., Ho, K.M., and Tringides, M.C., 2014. Nonclassical "Explosive" Nucleation in Pb/Si(111) at Low Temperatures, Phys. Rev. Lett. 113, 236201. https://doi.org/10.1103/PhysRevLett.113.236101. 
Figures and figure captions
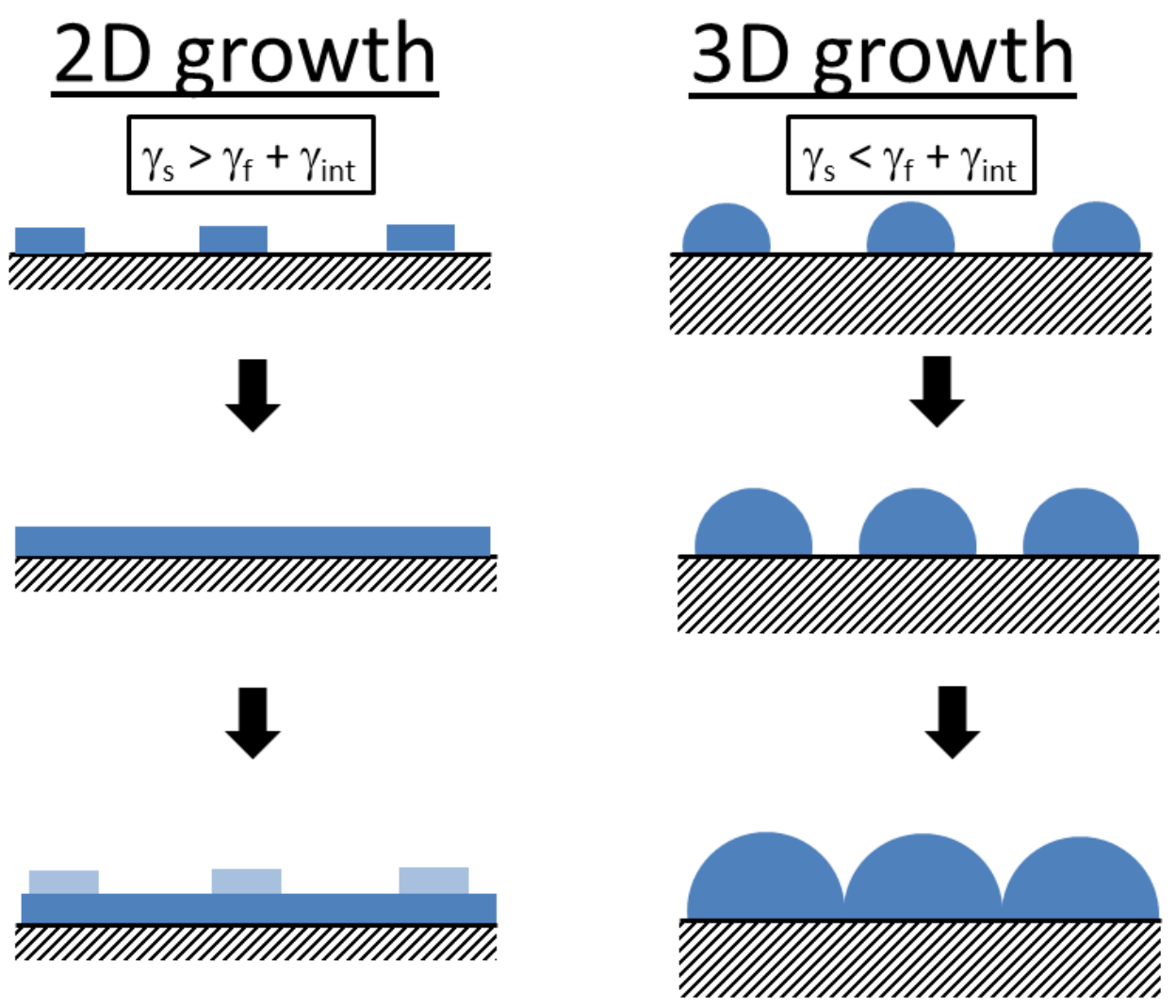

Fig. 1. Schematic illustration of $2 \mathrm{D}$ and $3 \mathrm{D}$ film growth according to the classification proposed by Bauer [3] 


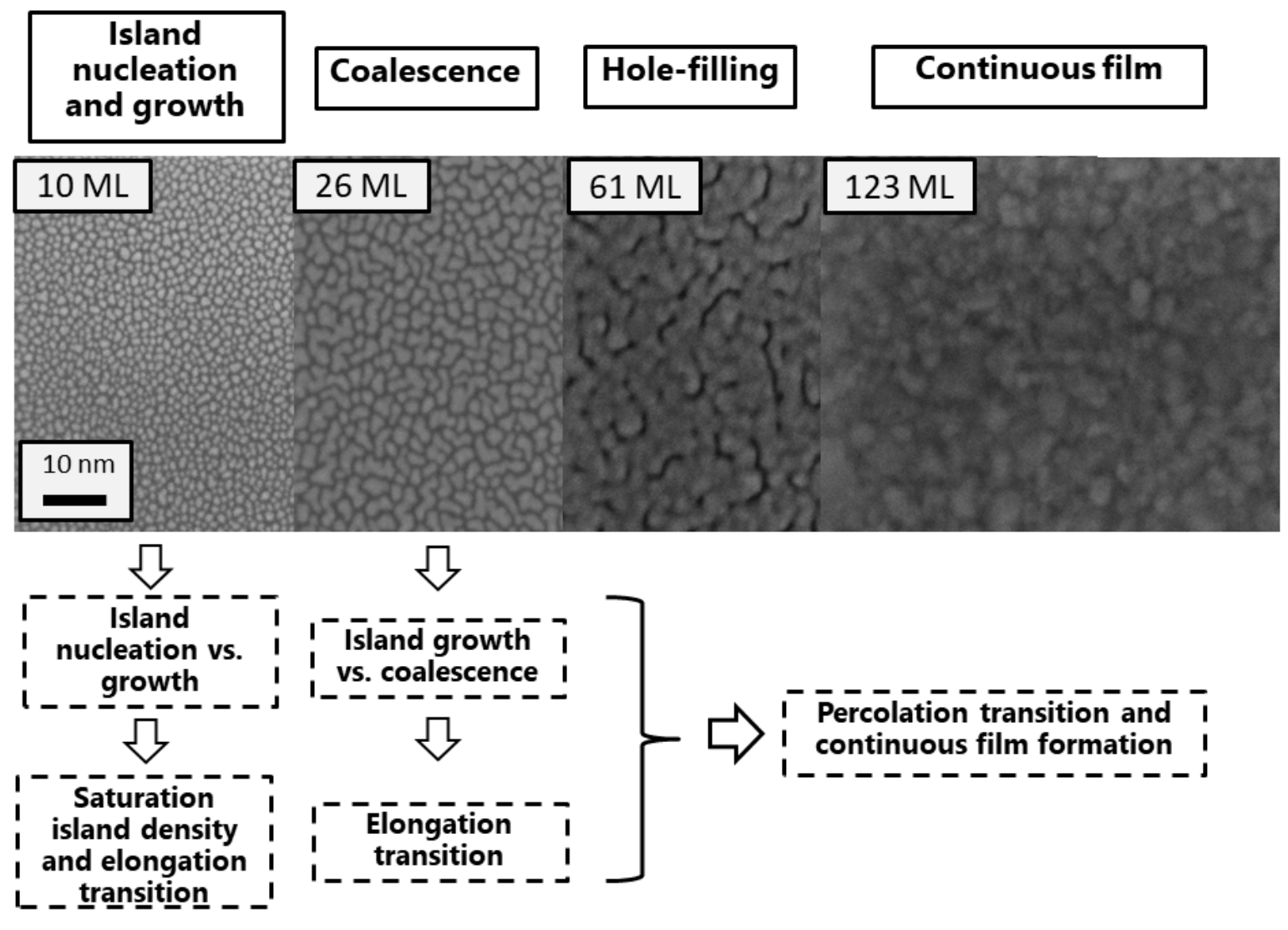

Fig. 2. Plan-view scanning electron microscopy images of magnetron sputtered $\mathrm{Ag}$ films grown on $\mathrm{SiO}_{2}$ substrates at various nominal thicknesses, which correspond to the different growth stages. The competing structure-forming processes that lead to saturation island density and elongation transition are also indicated in the figure. More details can be found in the text (Section 2). Nominal thicknesses are expressed in monoloayers (ML), whereby one monolayer is the (111) Ag interatomic distance, i.e., $2.35 \AA$ (Jamnig, Sarakinos, et al., unpublished data). 


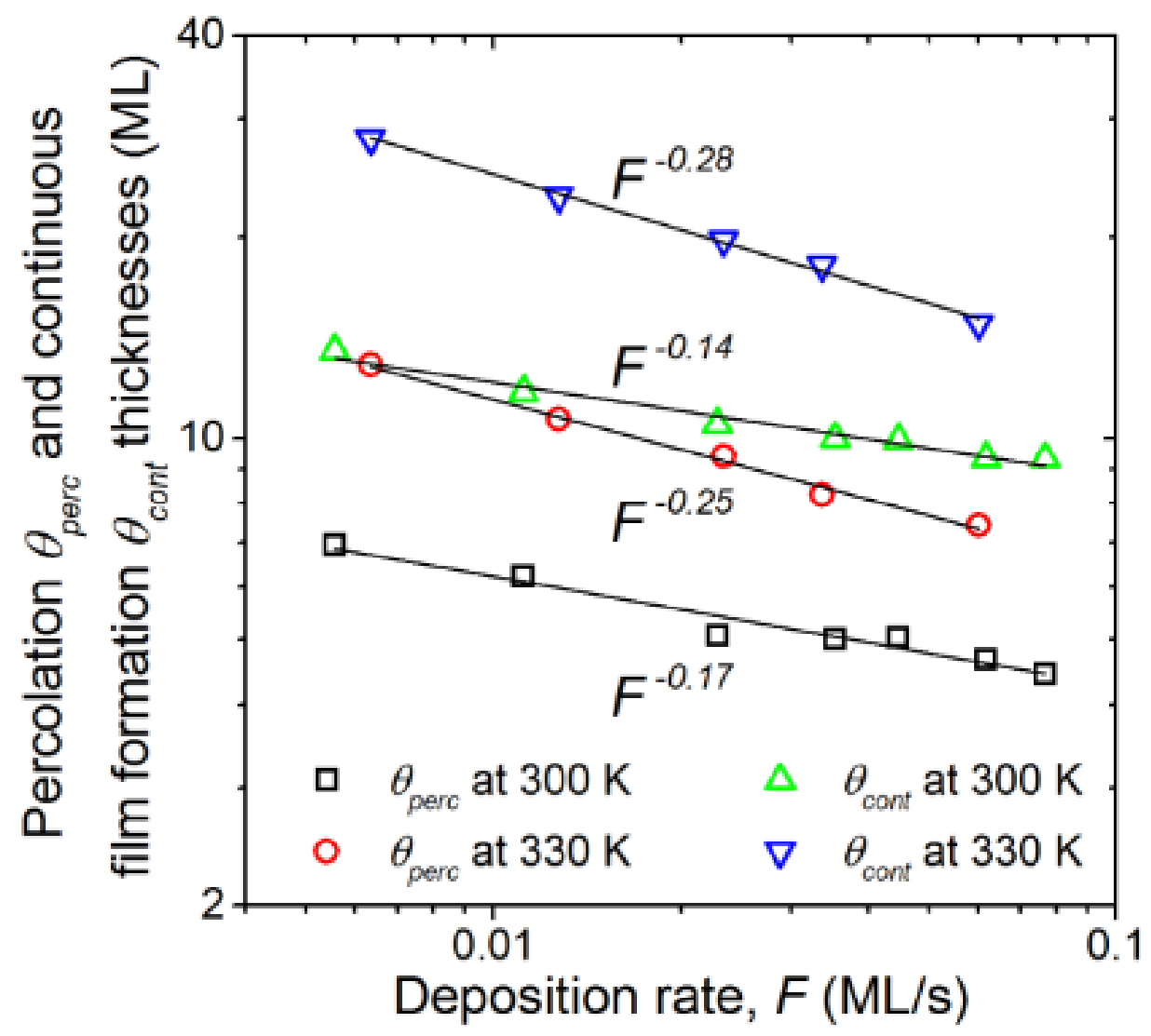

Fig. 3. Measured percolation transition $\Theta_{\text {perc }}$ and continuous film formation $\Theta_{\text {cont }}$ thicknesses for $\mathrm{Ag}$ films deposited on $\mathrm{SiO}_{2}$ substrates at room temperature (300 K) and elevated temperature $(330 \mathrm{~K})$ as a function of deposition rate $\mathrm{F}$. The data points are fitted with power-law expressions (lines) from which the exponents of $F$ are found. The scaling exponent for $300 \mathrm{~K}$ corresponds to Eq. (6) (i.e., coalescence-free growth), while the scaling exponent for $330 \mathrm{~K}$ is consistent with coalescence-controlled growth (Eq. (5)) (reprinted from Reference [35] with permission from the American Institute of Physics). 

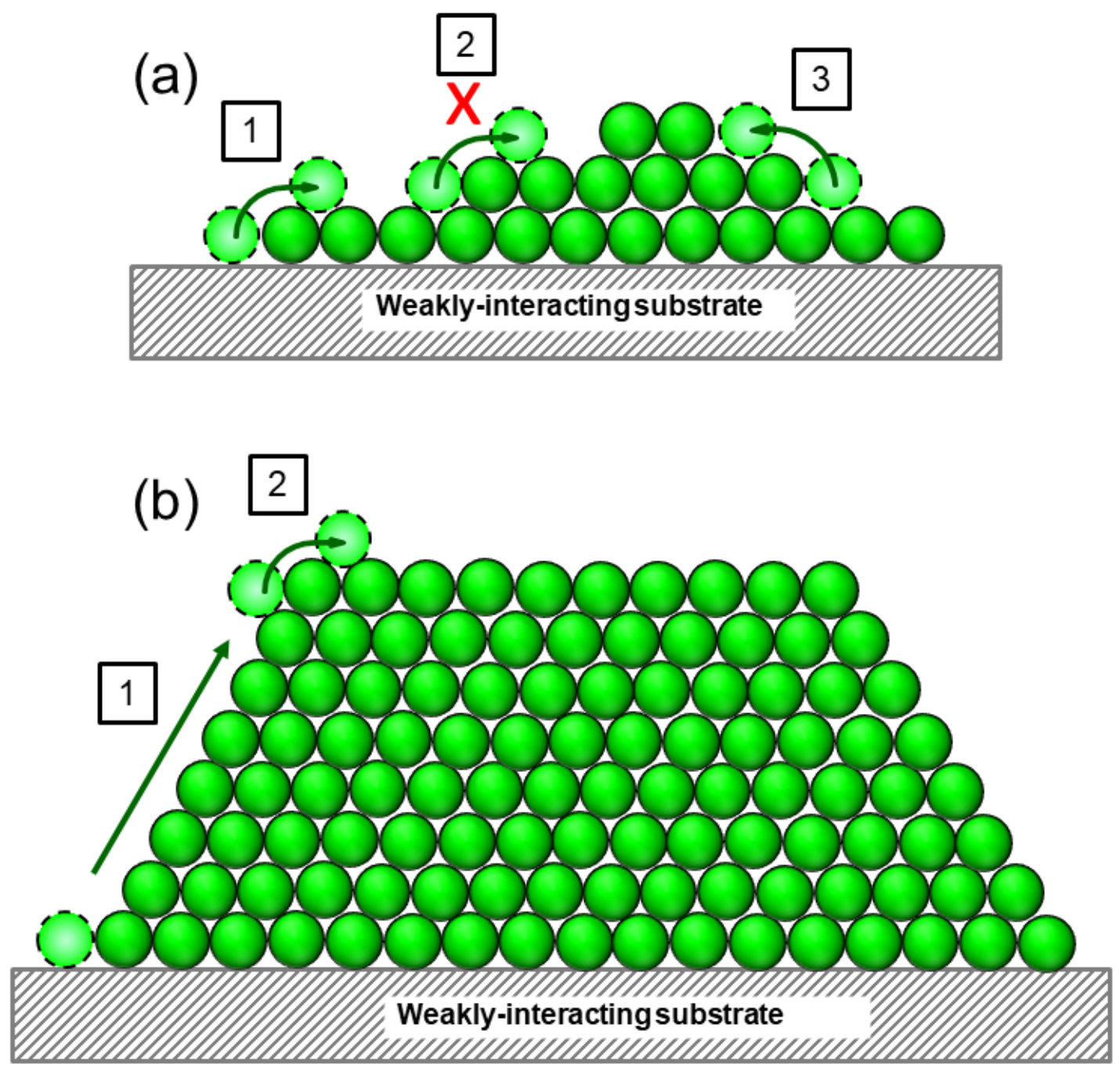

Fig. 4. Schematic illustrations of upward atomic transport mechanisms during 3D island formation on weakly-interacting substrates. (a) For an island that has a stepped structure, a weak adatom/substrate, vs adatom/film, interaction facilitates transport from the substrate onto the first island layer (process (1)), but the onset of homoepitaxial growth conditions on the first layer impose a larger barrier for an adatom crossing the atomic step to the second island layer (process (2)). The close horizontal proximity of atomic steps facilitates upward atomic transport onto the second island layer, via the assisted up-stepping mechanism (process (3)). (b) The formation of lowindex sidewall facets provides a facile diffusion pathway for upward mass transport (process (1)) and the rate-limiting step is the crossing of atoms from the sidewall facet 
to the top island layer (process (2). Solid spheres represent island atoms, while hollow spheres represent migrating adatoms. The diffusion direction is indicated by arrows, while "X" represents an energetically unfavorable pathway. 
(a)

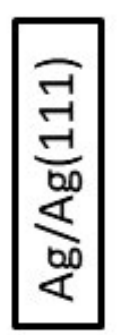

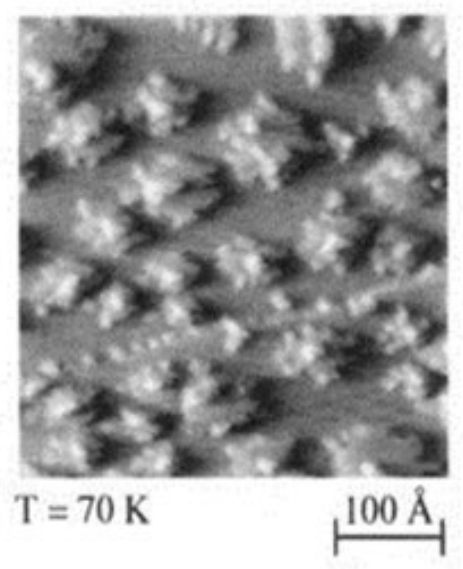

$$
\text { (c) } \mathrm{T}=298 \mathrm{~K}
$$

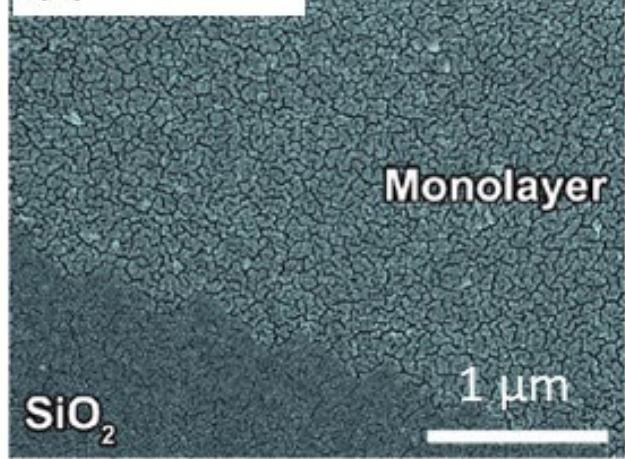

(b)
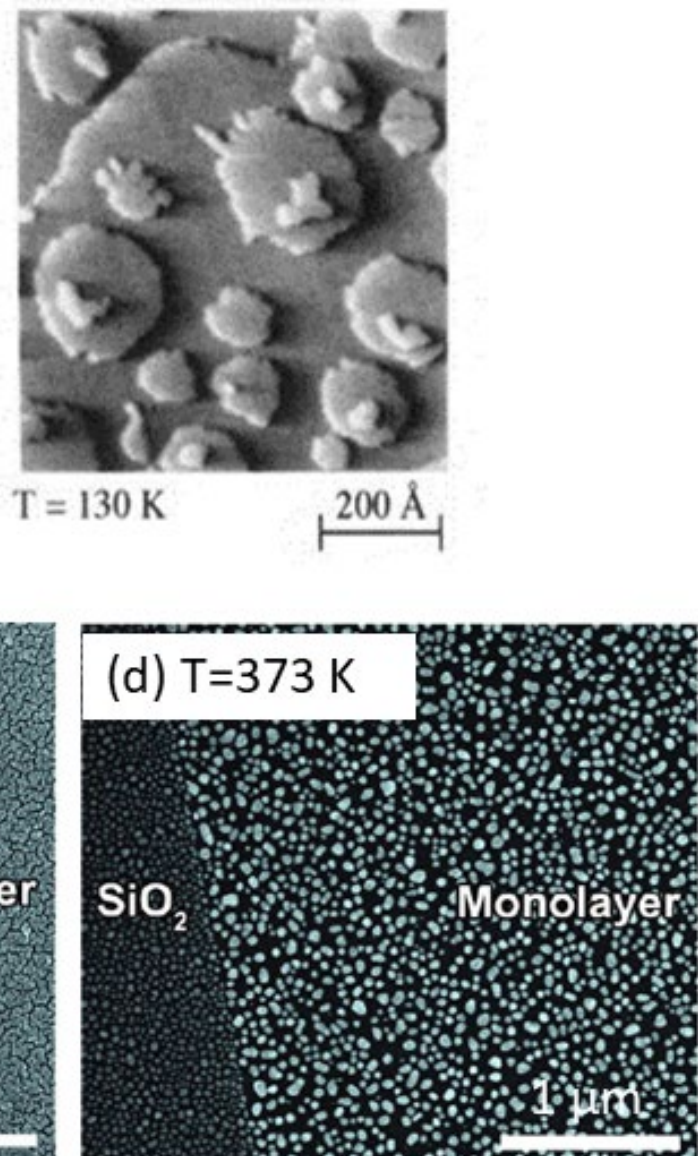

Fig. 5. Effect of growth temperature on morphology of (a) and (b) Ag films on Ag(111) substrates(reprinted from Reference [51] with permission from the American Physical Society); and (c) and (d) Ag films on graphene (reprinted from reference [50] with permission from ACS Publications). Ag on $\mathrm{Ag}(111)$ deposition has been performed using molecular beam epitaxy and morphology is imaged using scanning tunneling microscopy. Ag on graphene deposition has been performed by electron beam evaporation and the imaging is done by plan-view scanning electron microscopy. 